\title{
Regulation of plasma fibroblast growth factor 23 by calcium in primary hyperparathyroidism
}

Keisuke Kobayashi, Yasuo Imanishi, Akimitsu Miyauchi ${ }^{1}$, Naoyoshi Onoda ${ }^{2}$, Takehisa Kawata, Hideki Tahara, Hitoshi Goto, Takami Miki ${ }^{3}$, Eiji Ishimura, Toshitsugu Sugimoto ${ }^{4}$, Tetsuro Ishikawa ${ }^{5}$, Masaaki Inaba and Yoshiki Nishizawa

Department of Metabolism, Endocrinology and Molecular Medicine, Osaka City University Graduate School of Medicine 1-4-3, Asahi-machi, Abeno-ku, Osaka 545-8585, Japan, ${ }^{1}$ National Hyogo Chuo Hospital, Hyogo, Japan, ${ }^{2}$ Department of Oncology and ${ }^{3}$ Department of Geriatrics and Neurology, Osaka City University Graduate School of Medicine, Osaka, Japan, ${ }^{4}$ Division of Endocrinology, Metabolism, Hematology and Oncology, Department of Medicine, Shimane University School of Medicine, Shimane, Japan and ${ }^{5}$ Department of Surgical Oncology, Osaka City University Graduate School of Medicine, Osaka, Japan

(Correspondence should be addressed to Y Imanishi; Email: imanishi@med.osaka-cu.ac.jp)

\begin{abstract}
Objective: While the importance of fibroblast growth factor (FGF)-23 is established in phosphate-wasting disorders, little is known about the mechanisms regulating its circulating level. To investigate the role of parathyroid hormone (PTH) and calcium in FGF-23 metabolism, we examined plasma FGF-23 levels in patients with primary hyperparathyroidism (PHPT).

Patients and methods: Fifty patients with PHPT and 52 controls were employed in this study. Plasma was obtained from 18 PHPT patients who underwent parathyroidectomy (PTX) on the first postoperative morning without vitamin D administration. Time-course samples were also obtained from 5 of 18 PTX patients without vitamin D analogs or calcium administration. The expression of Fgf23 on resected parathyroid glands was analyzed by reverse transcription (RT)-PCR and immunohistochemistry.

Results: FGF-23 was significantly elevated in PHPT patients compared with controls. FGF-23 levels were significantly correlated positively with serum corrected calcium and intact PTH levels, and negatively with creatinine clearance and inorganic phosphate, among which creatinine clearance and corrected calcium were independently associated factors. In 18 PTX patients, postoperative FGF-23 levels were significantly decreased compared with preoperative levels. Corrected-calcium levels were significantly decreased $1 \mathrm{~h}$ after PTX, and this was followed by a reduction in plasma FGF-23 levels in time-course study. In addition, postoperative FGF-23 levels in 18 PTX patients were significantly correlated with corrected calcium, consistent with a role of serum calcium as one of the major regulators of FGF-23. The absence of Fgf 23 expression in parathyroid glands indicated that the parathyroid glands were not major sources of circulating FGF-23.

Conclusions: Serum calcium may regulate circulating FGF-23 levels in PHPT.
\end{abstract}

European Journal of Endocrinology 154 93-99

\section{Introduction}

Excess of fibroblast growth factor-23 (FGF-23), a member of the FGF family, is now known to be a major factor in hypophosphatemic rickets, such as X-linked hypophosphatemia (XLH) and oncogenic osteomalacia (OOM) (1-3). Autosomal dominant hypophosphatemic rickets (ADHR), a similar disorder characterized by renal phosphate wasting, has been reported to be associated with mutations of FGF-23 (4) that prevent its cleavage (5).

Administration of recombinant FGF-23 decreased serum phosphate levels in mice by an increase in renal phosphate excretion (6). Mice bearing a Chinese hamster ovary cell line stably expressing fgf 23 also showed hypophosphatemic rickets in vivo (6), indicating the importance of FGF-23 for hypophosphatemic rickets.

While the importance of FGF-23 is established in the pathogenesis of phosphate wasting disorders, the regulation of its plasma level is still controversial. Because of its hypophosphatemic effect, plasma FGF-23 levels may be regulated or affected by plasma phosphate level. Recently, it has been shown that plasma FGF-23 levels exhibited significant and positive correlations with serum parathyroid hormone (PTH), calcium and phosphate levels in uremic patients on maintenance hemodialysis $(7,8)$. FGF-23 levels were reported to be elevated significantly in humoral hypercalcemia of malignancy, and slightly, but not statistically 
significantly, elevated in patients with primary hyperparathyroidism (PHPT) (9).

Recently, elevated FGF-23 levels were observed in PHPT patients compared with control subjects (10). Creatinine clearance and serum calcium were promoted to be independently associated factors by multiple-regression analysis; however, the FGF-23 levels did not change by parathyroidectomy (PTX) despite the normalization of serum calcium and PTH levels. The authors suggested that circulating FGF-23 was not influenced by serum calcium (10). In the present study, we attempted to determine the role of serum calcium in the regulation of FGF-23 levels in patients with PHPT. Without loading of vitamin D analogs, which were recently demonstrated to increase serum FGF-23 levels (11), FGF-23 levels were significantly decreased on the morning after PTX. In addition, postoperative FGF-23 levels in PTX patients were significantly correlated with corrected serum calcium, consistent with a role of serum calcium as a major regulator of FGF-23. These data suggest that serum calcium is a regulator of circulating FGF-23 levels.

\section{Materials and methods}

\section{Patients}

This study included 50 patients with PHPT and 52 healthy control subjects. Subjects were restricted to those with serum creatinine less than $100 \mu \mathrm{mol} / \mathrm{l}$. All subjects provided written, informed consent before participation in this study, which was approved by institutional ethics committees (Osaka City University Graduate School of Medicine, National Hyogo Chuo Hospital, and Kobe University Graduate School of Medicine) and was conducted in accordance with the principles of the Declaration of Helsinki.

Forty-one of 50 PHPT patients underwent PTX, and 38 of 41 PTX patients were diagnosed with parathyroid adenoma, and three with parathyroid hyperplasia. No parathyroid carcinoma was included in this study. Among them, pre- and postoperative samples were obtained from 18 PTX patients who received PTX between October 2002 and September 2003 in Osaka City University Hospital. They were all diagnosed with parathyroid adenoma. Time-course samples were obtained from the five most recent cases of the 18 PTX patients, whose PTX were performed between February 2003 and September 2003. No significant difference was found between all the PHPT patients, the 18 PTX patients and the selected five PTX time-course patients in pre-PTX levels of serum calcium $(2.79 \pm 0.02, \quad 2.77 \pm 0.05$ and $2.84 \pm 0.10 \mathrm{mmol} / \mathrm{l}$

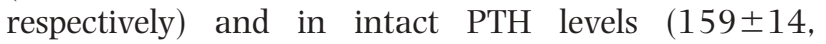
$179 \pm 35$ and $222 \pm 54 \mathrm{ng} / \mathrm{l}$ respectively).

The control subjects were randomly selected from our database after categorization by age range and gender, so that these two groups were comparable for age and gender.

\section{Measurement of plasma FGF-23 concentration}

Blood samples were obtained from all PHPT patients and healthy volunteers after overnight fast. The blood was quickly mixed with edetic acid, and plasma was separated by centrifugation. Plasma samples were stored at $-20{ }^{\circ} \mathrm{C}$ until assay. Plasma FGF-23 concentrations were measured with the human FGF-23 (C-Term) ELISA kit (Immutopics Inc., San Clemente, CA, USA) according to the manufacturer's instructions. Serum samples were also collected at the same time to measure other parameters.

\section{Time course of plasma FGF-23 levels and parameters related to calcium metabolism}

Pre- and postoperative samples were collected from 18 PHPT patients who received therapeutic PTX. Preoperative samples were obtained on the morning of the day of PTX $(0700 \mathrm{~h})$, and postoperative samples on the morning of the next day $(0700 \mathrm{~h})$. None had received bisphosphonates or vitamin D analogs before PTX. All samples were fasting samples. Treatment of hypocalcemia after PTX was performed by intravenous administration of calcium gluconate. The amount of calcium gluconate administered was up to $235.5 \mathrm{mg}$ between the end of the operation and the next morning. No patients were administered a vitamin $\mathrm{D}$ analog.

Five of 18 PHPT patients who received PTX were further examined for the time course of changes in plasma FGF-23 levels after PTX. Plasma and serum samples were collected before PTX and at set times after the operation. Neither vitamin D analogs nor calcium gluconate was administered during the timecourse study.

\section{Fgf 23 expression in parathyroid glands}

To determine whether the parathyroid glands express Fgf23, reverse transcription (RT)-PCR and immunohistochemical analyses were performed on the excised glands. A survey for expression of the Fgf 23 gene was performed by RT-PCR analysis with primers that reside in exons 1 and 3 of Fgf 23 (sense primer: 5'-CACAGCCACAGCCAGGAACAGC-3'; antisense primer: $5^{\prime}$ GTCGTACCCGTTTTCCAGCGTCT-3'). Immunohistochemical analysis was performed with goat antihFGF23 (225-244) antibody (Immutopics), followed by visualization with the Vectastain $\mathrm{ABC}$ kit (Vector Laboratories, Ingold Road Burlingame, CA). The slides were counterstained with Mayer's hematoxylin. OOM tumors expressing Fgf 23 were used as positive controls. 


\section{Statistical analysis}

Results were denoted as mean \pm S.E. Serum calcium levels were expressed as corrected calcium after adjustment for serum albumin. Correlations between two variables were examined by Spearman's correlation coefficient and linear regression analysis. The relationship between several clinical variables and log FGF-23 was investigated by multiple-regression analysis. Because intact PTH and FGF-23 exhibited skewed distributions, log-transformed values were used to fit linear models. Differences in FGF-23 levels between preoperative and postoperative samples were evaluated by Wilcoxon's signed-rank test. Time-course changes in parameters were analyzed by repeated-measure oneway analysis of variance. $P$ values less than 0.05 were considered statistically significant. Furthermore, we confirmed the results as statistically significant by looking at the change from baseline at each time point. All analyses were performed with commercially available software for Windows (StatView V; SAS Institute, Cary, NC, USA).

\section{Results}

\section{Plasma FGF-23 levels and other serum parameters}

This study used 50 PHPT patients and 52 healthy controls to investigate which parameters are involved in the regulation of plasma FGF-23 levels in PHPT. The PHPT patients enrolled in the present study revealed hypercalcemia, hypophosphatemia and elevated serum intact PTH and 1,25(OH)2D levels, compared with controls, consistent with the diagnosis of PHPT (Table 1). Plasma FGF-23 levels were significantly elevated in PHPT compared with controls (Table 1).

\section{Correlations between plasma FGF-23 levels and other variables}

The correlations of these variables with plasma FGF-23 levels were examined to determine which parameters are important determinants of plasma FGF-23. Plasma FGF-23 level was significantly correlated in a positive manner with corrected calcium (from normoto hypercalcemia), inorganic phosphate and intact PTH, but not with serum $1,25(\mathrm{OH}) 2 \mathrm{D}$, by correlation coefficient analyses (Table 2). The same results were obtained by simple regression analysis when intact PTH and FGF-23 were log-transformed, since the subjects as a whole (PHPT patients plus healthy controls) revealed normal distributions of log serum intact PTH, log FGF-23 and other serum variables (Table 2). Furthermore, although subjects were restricted to those with serum creatinine less than $100 \mu \mathrm{mol} / \mathrm{l}$, plasma FGF-23 correlated significantly with creatinine clearance.

\section{Factors independently associated with log plasma FGF-23 levels}

Factors independently associated with log FGF-23 level were examined with a multiple-regression model. Log FGF-23 exhibited significant and independent associations with creatinine clearance and corrected calcium (Table 3). However, serum inorganic phosphate and $1,25(\mathrm{OH}) 2 \mathrm{D}$ levels failed to emerge as significant factors in these models.

\section{Time course of changes in serum parameters after PTX}

To examine the effect of PTH on FGF-23 levels, the levels were measured on the morning of PTX and on the morning after PTX. No patients were administered a vitamin D analog until the morning after PTX. The FGF-23 level was significantly decreased from $94.0 \pm 12.6$ relative units $(\mathrm{RU}) / \mathrm{ml}$ to $78.2 \pm 12.1$ $(P=0.0429$ by Wilcoxon's signed-rank test) (Fig. 1). The FGF-23 level 7 days after PTX was $85.4 \pm 22.7 \mathrm{RU} / \mathrm{ml}$, which was not significant in relation to preoperative data $(P=0.7671)$.

To examine further the role of serum parameters in the determination of plasma FGF-23, the time course of changes in plasma FGF-23 levels was monitored until the day after PTX. Five PHPT patients were studied. Neither vitamin D analogs nor calcium gluconate was administered during the time-course study.

Table 1 Clinical details of subjects.

\begin{tabular}{|c|c|c|c|c|}
\hline & Normal range & PHPT & Control & $P$ \\
\hline$n$ & & 50 & 52 & \\
\hline Age (years) & & $61.4 \pm 1.6$ & $62.2 \pm 2.7$ & 0.111 \\
\hline Creatinine clearance $(\mathrm{ml} / \mathrm{s})$ & $1.17-2.00$ & $1.39 \pm 0.06$ & $1.34 \pm 0.08$ & 0.393 \\
\hline Corrected calcium (mmol/l) & $2.20-2.54$ & $2.79 \pm 0.02$ & $2.40 \pm 0.02$ & $<0.001$ \\
\hline Inorganic phosphate $(\mathrm{mmol} / \mathrm{l})$ & $0.94-1.39$ & $0.86 \pm 0.02$ & $1.10 \pm 0.23$ & $<0.001$ \\
\hline Intact PTH (ng/l) & $10-65$ & $159 \pm 14$ & $46 \pm 3$ & $<0.001$ \\
\hline $1,25(\mathrm{OH})^{2} \mathrm{D}(\mathrm{pmol} / \mathrm{l})$ & $71-179$ & $177 \pm 8$ & $151 \pm 8$ & 0.027 \\
\hline FGF-23 (RU/ml) & $0-155$ & $111 \pm 17$ & $61 \pm 4$ & $<0.001$ \\
\hline
\end{tabular}

Values represent means \pm S.E.M. $P$ values calculated by Mann-Whitney U test. PHPT: primary hyperparathyroidism. RU: relative units. 
Table 2 Serum variables affecting plasma FGF-23 levels.

\begin{tabular}{lrrrrr}
\hline & \multicolumn{2}{c}{ Spearman's } & & \multicolumn{2}{c}{$\begin{array}{c}\text { Simple } \\
\text { regression }\end{array}$} \\
\cline { 2 - 3 } \cline { 6 - 7 } Variables & \multicolumn{1}{c}{$\rho$} & \multicolumn{1}{c}{$P$} & & $r$ & $P$ \\
\hline Creatinine clearance & -0.274 & 0.006 & & -0.279 & 0.005 \\
Corrected calcium & 0.409 & $<0.001$ & & 0.356 & $<0.001$ \\
Inorganic phosphate & -0.247 & 0.013 & & -0.218 & 0.028 \\
Intact PTH & 0.298 & 0.003 & & 0.264 & 0.008 \\
$1,25(\mathrm{OH})^{2} \mathrm{D}$ & -0.028 & 0.804 & & 0.055 & 0.634 \\
\hline
\end{tabular}

Fifty PHPT and 52 healthy controls were employed in this analysis. Spearman's correlation coefficient with serum variables as independent variables versus plasma FGF-23 as dependent variables was analyzed in the subjects. Simple-regression analyses were performed after log-transformation of intact PTH and FGF-23, because these variables had skewed distributions.

The changes in serum intact PTH and corrected calcium, and plasma FGF-23 were significant after PTX (Table 4 and Fig. 2). Neither serum phosphate nor $1,25(\mathrm{OH}) 2 \mathrm{D}$ changed significantly during this period. These parameters attained steady-state levels $6 \mathrm{~h}$ after PTX. Plasma FGF-23 levels were decreased significantly at $6 \mathrm{~h}$, and the levels were essentially unchanged on the morning after PTX (Fig. 2). The degree to which plasma FGF-23 decreased was similar to that of the correctedcalcium level (FGF-23; 91\%, 85\%, 68\% and 70\%, corrected calcium; $90 \%, 86 \%, 86 \%$ and $79 \%$, at $1 \mathrm{~h}, 3 \mathrm{~h}$, $6 \mathrm{~h}$ and next day after PTX respectively).

\section{Correlation between plasma FGF-23 and corrected-calcium level on the day after PTX}

In the 18 patients who underwent PTX, FGF-23 and corrected calcium attained steady-state levels on the day after PTX. FGF-23 was significantly correlated positively with corrected calcium on the next morning of PTX $(r=0.598, P=0.0137)$ (Fig. 3). A positive

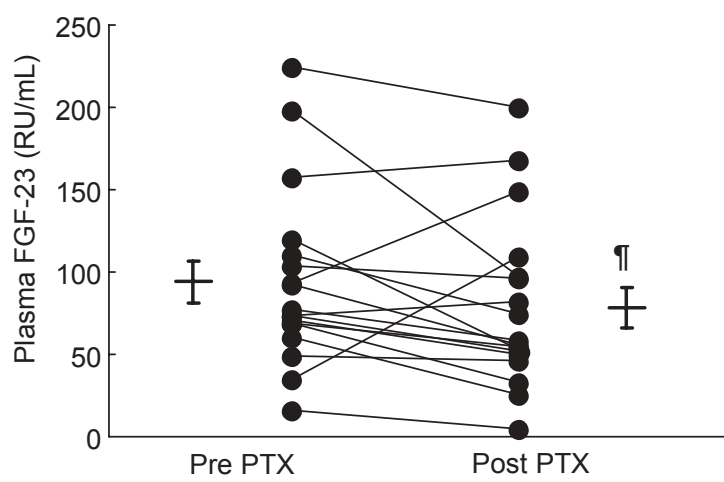

Figure 1 Changes in plasma FGF-23 levels in PHPT patients after PTX. Pre- and postoperative samples were obtained from 18 PHPT patients who received PTX. Preoperative samples were obtained on the morning of the day of PTX $(0700 \mathrm{~h})$, and postoperative samples on the morning of the next day $(0700 \mathrm{~h})$. Values represent means \pm S.E.M. " $P=0.0429$, by Wilcoxon's signed-rank test. $\mathrm{RU}$ : relative units.
Table 3 Multiple-regression analysis of factors affecting plasma log FGF-23 levels.

\begin{tabular}{lrc}
\hline Variables & $\boldsymbol{\beta}$ & $\boldsymbol{P}$ \\
\hline Creatinine clearance & -0.253 & 0.0085 \\
Corrected calcium & 0.335 & 0.0126 \\
Inorganic phosphate & -0.025 & 0.8421 \\
Log intact PTH & -0.003 & 0.9836 \\
& $R^{2}=0.195$ & 0.0003
\end{tabular}

Fifty PHPT and 52 healthy controls were employed in this analysis. The table lists standard regression coefficients ( $\beta$ values) and levels of significance.

correlation existed between delta FGF-23 (FGF-23 on the next morning - FGF-23 pre-PTX) and the dose of calcium gluconate administration during PTX and the next morning ( $\rho=0.438, P=0.0709$, by correlation coefficient) at borderline significance (data not shown).

\section{Fgf-23 expression in parathyroid glands by RT-PCR and immunohistochemistry}

Neither RT-PCR nor immunohistochemistry revealed the expression of Fgf-23 in surgically removed parathyroid glands. Positive controls showed Fgf-23 expression by both methods (Fig. 4).

\section{Discussion}

The present study demonstrated plasma FGF-23 levels to be significantly higher in PHPT patients than in controls. We (7) and others (8) have reported that plasma FGF-23 levels are significantly raised in uremic patients on maintenance hemodialysis and are significantly and independently correlated in a positive manner with serum calcium, phosphate and PTH. These data, together with the concept that FGF-23 is a

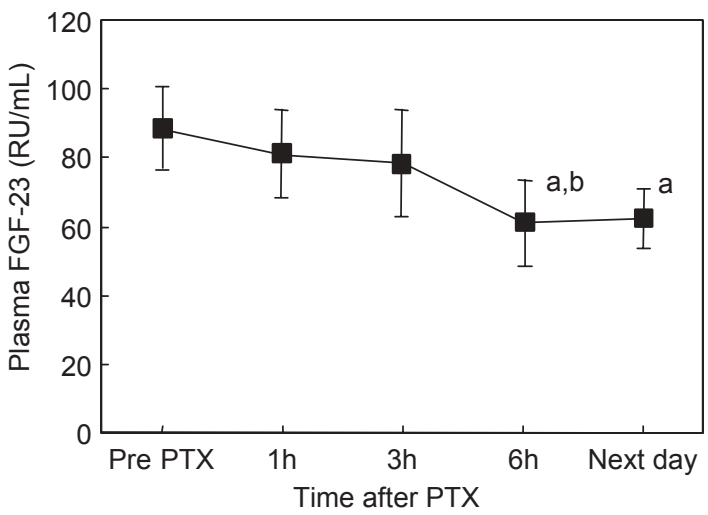

Figure 2 Time course of changes in plasma FGF-23 levels in five PHPT patients after PTX. Changes in plasma FGF-23 levels were significant $(P=0.031)$ by repeated-measures one-way analysis of variance. Values represent means \pm S.E.M. ${ }^{\mathrm{a}} P<0.05$ vs pre-PTX; ${ }^{\mathrm{b}} P<0.05$ vs $1 \mathrm{~h}$ by Fisher's protected least significant difference. $\mathrm{RU}$ : relative units. 
Table 4 Time course of changes in serum parameters during PTX.

\begin{tabular}{lccccccc}
\hline & & \multicolumn{3}{c}{ Time after PTX } & \\
\cline { 3 - 6 } & Normal range & Pre-PTX & $1 \mathrm{~h}$ & $3 \mathrm{~h}$ & $6 \mathrm{~h}$ & Next day & $\boldsymbol{P}$ \\
\hline Intact PTH $(\mathrm{ng} / \mathrm{l})$ & $10-65$ & $222 \pm 54$ & $22 \pm 4^{\mathrm{a}}$ & $14 \pm 3^{\mathrm{a}}$ & $13 \pm 3^{\mathrm{a}}$ & $21 \pm 11^{\mathrm{a}}$ & $<0.001$ \\
Corrected calcium $(\mathrm{mmol} / \mathrm{l})$ & $2.20-2.54$ & $2.84 \pm 0.12$ & $2.57 \pm 0.12^{\mathrm{a}}$ & $2.45 \pm 0.10^{\mathrm{a}, \mathrm{b}}$ & $2.42 \pm 0.07^{\mathrm{a}, \mathrm{b}}$ & $2.25 \pm 0.10^{\mathrm{abcd}}$ & $<0.001$ \\
Inorganic phosphate $(\mathrm{mmol} / \mathrm{l})$ & $0.94-1.39$ & $0.84 \pm 0.03$ & $0.87 \pm 0.03$ & $0.90 \pm 0.10$ & $1.03 \pm 0.10$ & $0.94 \pm 0.13$ & 0.161 \\
$1,25(\mathrm{OH})^{2} \mathrm{D}(\mathrm{pmol} / \mathrm{l})$ & $71-179$ & $273 \pm 42$ & $161 \pm 35$ & $207 \pm 44$ & $256 \pm 58$ & $188 \pm 32$ & 0.052 \\
\hline
\end{tabular}

Values are representing mean \pm S.E.M. $P$ values of changes in serum parameters were calculated by repead-measures one-way analysis of variance.

${ }^{\mathrm{a}} \mathrm{P}<0.05$ vs Pre-PTX; ${ }^{\mathrm{b}} \mathrm{P}<0.05$ vs $1 \mathrm{~h} ;{ }^{\mathrm{c}} \mathrm{P}<0.05$ vs $3 \mathrm{~h} ;{ }^{\mathrm{d}} \mathrm{P}<0.05$ vs 6 h by Fisher's protected least significant difference.

major determinant of phosphate metabolism, suggest the presence of a regulatory effect by serum phosphate on plasma FGF-23 levels. Since these patients retain extraordinary amounts of phosphate, the regulation by serum phosphate may be pathologic but not physiologic. In the present study, we tried to elucidate the major determinant of plasma FGF-23 levels in all patients (healthy controls and PHPT patients), whose serum phosphate levels did not differ significantly from those of control subjects. The subjects as a whole showed normal distributions of creatinine clearance, serum corrected calcium, phosphate, log intact PTH and log plasma FGF-23. Levels of plasma FGF-23 correlated with creatinine clearance, corrected calcium, inorganic phosphate and intact PTH by both parametric and nonparametric analyses. Plasma FGF-23 correlated significantly with creatinine clearance by both simple- and multiple-regression analyses. These data agree with the previous report on patients with PHPT (10). It is supposed that there is a tendency to retain phosphate even in mild renal insufficiency, and, to compensate for the transient phosphate retention, PTH and probably FGF-23 also increase

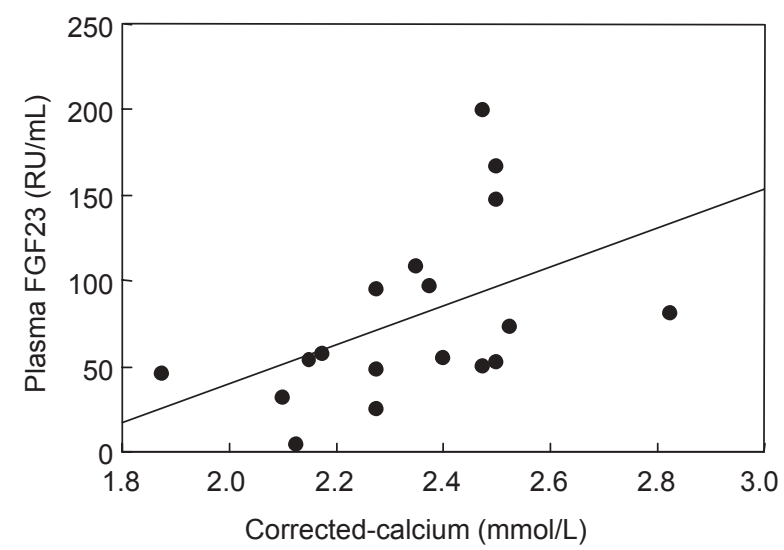

Figure 3 Simple regression analysis of plasma FGF-23 and corrected calcium levels in post-PTX subjects. Samples were collected from 18 PHPT patients on the morning after PTX. The FGF-23 levels were significantly correlated with corrected-calcium levels $(r=0.598, P=0.0137)$. RU: relative units. subsequently, leading to a reduction of renal phosphate reabsorption.

At $0700 \mathrm{~h}$ on the day after PTX, by which time serum corrected calcium had attained a steady-state level after the sudden withdrawal of PTH due to PTX, the level of corrected calcium was related to that of plasma FGF-23 (Fig. 2). Sequential observations of serum corrected-calcium and phosphate and plasma FGF-23 levels showed a reduction in serum corrected calcium at $1 \mathrm{~h}$ and a significant reduction in plasma FGF-23 level by $6 \mathrm{~h}$ after PTX. Serum phosphate increased after PTX, but not significantly. Taken together, these data suggest a role of serum calcium in the regulation of plasma FGF-23 levels in the subjects examined. The serum PTH levels among PHPT patients and healthy controls were distributed widely above the normal lower limit but did not include cases of hyperphosphatemia resulting from renal dysfunction. Since our previous study (7) elucidated the importance of serum phosphate in the regulation of plasma FGF-23 levels in uremic patients, control of plasma FGF-23 levels by serum phosphate may occur specifically in conditions of phosphate retention. Supporting this notion is a previous study indicating a lack of consistent effects of oral phosphate loading on FGF-23 levels in healthy volunteers (8). In uremic patients on maintenance hemodialysis, both corrected-calcium and intact PTH levels were significantly and independently associated with plasma FGF-23 levels $(7,8)$.

In this study, a significant association between corrected calcium and plasma FGF-23 was observed in the morning after PTX, when intrinsic PTH secretion was essentially deficient. In addition, delta FGF-23 also tended to correlate positively with the dose of calcium gluconate, supporting the hypothesis that serum calcium is a regulator of plasma FGF-23 level independently of serum PTH. Interestingly, plasma FGF-23 levels of 18 PTX patients in the morning significantly decreased from preoperative levels. The attenuated decrease of FGF-23 levels after PTX could be explained by intravenous injections of calcium gluconate after PTX to prevent tetany. These injections elevated both corrected-calcium and FGF-23 levels after PTX. It has been reported that plasma FGF-23 levels are increased 


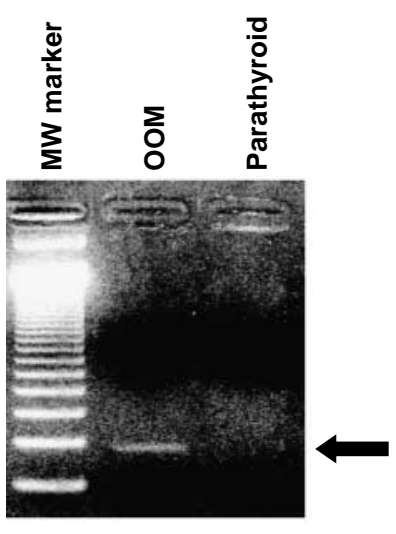

A

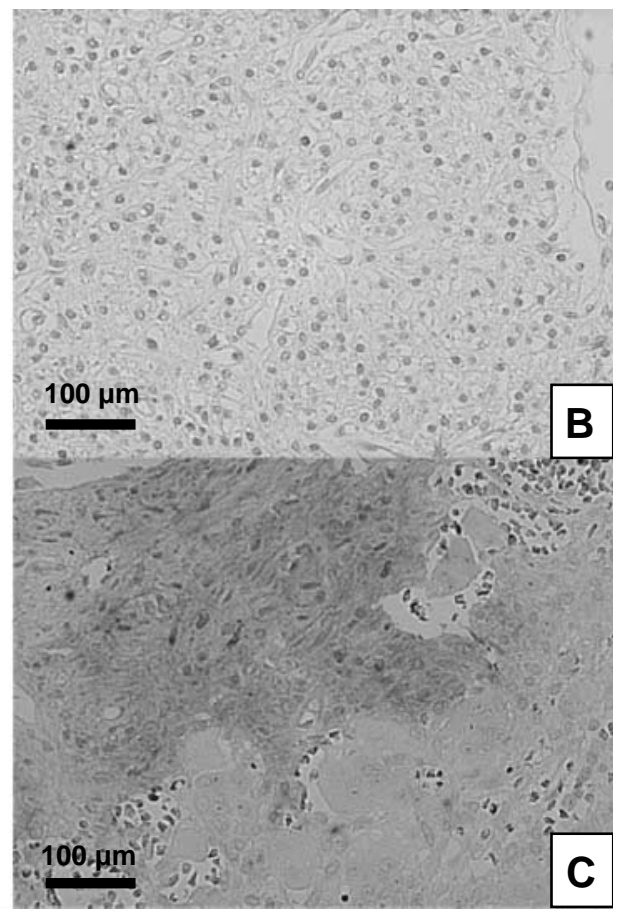

Figure 4 Expression analysis of Fgf23 in parathyroid glands by RT-PCR and immunohistochemistry. RT-PCR analysis of RNA from a tumor causing OOM with intron-spanning primers revealed a 226 bp Fgf23 product. Surgically resected parathyroid glands were negative for FGF-23 expression (A). Surgically resected parathyroid glands were negative for Fgf23 staining by immunohistochemical analysis (B), compared with the abundant expression in the tumor causing $\mathrm{OOM}(\mathrm{C})$. in patients with humoral hypercalcemia of malignancy (9), suggesting an effect either of increased serum PTHrelated peptide or of calcium, which may therefore have the same effect on plasma FGF-23 levels in these circumstances as in PHPT. The level of serum $1,25(\mathrm{OH}) 2 \mathrm{D}$ is also reported to correlate with FGF-23 in maintenance hemodialysis patients (8). In this study, 1,25(OH)2D level was not correlated with FGF23 in nonuremic patients, because the range of $1,25(\mathrm{OH}) 2 \mathrm{D}$ level was very narrow. Some of the PTX patients started to be administered oral 1,25(OH)2D3 to prevent tetany after the next morning of PTX. Decrement of circulating FGF-23 in the next morning of PTX and the attenuation of its decrement for 6 weeks after PTX was reported previously, although these changes were not statistically significant (12). The trend of the changes in FGF-23 was similar to the present study. In this and previous $(10,12)$ studies, this reversal of plasma FGF-23 levels to pre-PTX levels on several days after PTX could be explained by either the administration of $1,25(\mathrm{OH}) 2 \mathrm{D} 3$ to prevent tetany or mild reelevation of intrinsic PTH after PTX.

Although Fgf 23 mRNA expression has been reported in thyroid/parathyroid biopsy sections, as well as in heart and liver by RT-PCR (4), the major source of circulating FGF-23 cannot be secretion from the parathyroid glands, because disappearance of plasma FGF-23 from the circulation after PTX was incomplete compared with the decrement of plasma intact PTH level after PTX. In OOM, a decreased circulating FGF-23 level was already observed at $30 \mathrm{~min}$ after resection of the responsible tumor, and it had returned to a basal level at $60 \mathrm{~min}$ (3). This observation was confirmed by RT-PCR and immunohistochemical analysis of FGF-23 demonstrating the absence of Fgf23 expression in parathyroid glands obtained at PTX. Bone could be the source of FGF-23, explaining the increase in circulating FGF-23 in PHPT, because Fgf23 expression has been demonstrated by RT-PCR of human bone (data not shown), and bone is a major organ of the PTH-calcium regulatory system. Further studies should be performed on the pathophysiologic role of bone tissue in the regulation of plasma FGF-23. It would also be interesting to investigate the role of elevated FGF-23 in PHPT. An effect of reduction in serum phosphate by FGF-23 additional to the effect of PTH may prevent ectopic calcification by lowering the serum calcium $\mathrm{x}$ phosphate product.

In conclusion, it has been demonstrated that serum calcium may be one of regulators of circulating levels of FGF-23 independently of PTH in those without phosphate retention, in contrast to the role of serum phosphate in patients with phosphate retention. Our results provide new insights into the pathophysiologic effects of FGF-23 on calcium-phosphate homeostasis.

\section{Acknowledgements}

We thank Sumitomo Seiyaku Biomedical Co., Ltd for technical assistance in measurement of plasma FGF-23, Dr Jeffrey Lavigne (Immutopics, Inc.) for 
providing us with the goat anti-hFGF-23 antibody and Prof. Nobuyuki Itoh (Kyoto University) for providing us with human Fgf23 cDNA. This work was supported by a research grant from the Renal Osteodystrophy Foundation (Tokyo).

\section{References}

1 Jonsson KB, Zahradnik R, Larsson T, White KE, Sugimoto T, Imanishi Y, Yamamoto T, Hampson G, Koshiyama H, Ljunggren O, Oba K, Yang IM, Miyauchi A, Econs MJ, Lavigne J \& Juppner H. Fibroblast growth factor 23 in oncogenic osteomalacia and X-linked hypophosphatemia. New England Journal of Medicine 2003348 1656-1663.

2 White KE, Jonsson KB, Carn G, Hampson G, Spector TD, Mannstadt M, Lorenz-Depiereux B, Miyauchi A, Yang IM, Ljunggren O, Meitinger T, Strom TM, Juppner H \& Econs MJ. The autosomal dominant hypophosphatemic rickets (ADHR) gene is a secreted polypeptide overexpressed by tumors that cause phosphate wasting. Journal of Clinical Endocrinology and Metabolism 200186 497-500.

3 Yamazaki Y, Okazaki R, Shibata M, Hasegawa Y, Satoh K, Tajima T, Takeuchi Y, Fujita T, Nakahara K, Yamashita T \& Fukumoto S. Increased circulatory level of biologically active full-length FGF-23 in patients with hypophosphatemic rickets/osteomalacia. Journal of Clinical Endocrinology and Metabolism 2002 87 4957-4960.

4 ADHR Consortium. Autosomal dominant hypophosphataemic rickets is associated with mutations in FGF23. Nature Genetics 200026 345-348.

5 White KE, Carn G, Lorenz-Depiereux B, Benet-Pages A, Strom TM \& Econs MJ. Autosomal-dominant hypophosphatemic rickets (ADHR) mutations stabilize FGF-23. Kidney International 2001 $602079-2086$.
6 Shimada T, Mizutani S, Muto T, Yoneya T, Hino R, Takeda S, Takeuchi Y, Fujita T, Fukumoto S \& Yamashita T. Cloning and characterization of FGF23 as a causative factor of tumor-induced osteomalacia. PNAS 200198 6500-6505.

7 Imanishi Y, Inaba M, Nakatsuka K, Nagasue K, Okuno S, Yoshihara A, Miura M, Miyauchi A, Kobayashi K, Miki T, Shoji T, Ishimura E \& Nishizawa Y. FGF-23 in patients with end-stage renal disease on hemodialysis. Kidney International 200465 1943-1946.

8 Larsson T, Nisbeth U, Ljunggren O, Juppner H \& Jonsson KB. Circulating concentration of FGF-23 increases as renal function declines in patients with chronic kidney disease, but does not change in response to variation in phosphate intake in healthy volunteers. Kidney International $2003642272-2279$.

9 Singh RJ \& Kumar R. Fibroblast growth factor 23 concentrations in humoral hypercalcemia of malignancy and hyperparathyroidism. Mayo Clinical Proceedings 2003 78 826-829.

10 Yamashita H, Yamashita T, Miyamoto M, Shigematsu T, Kazama JJ, Shimada T, Yamazaki Y, Fukumoto S, Fukagaw M \& Noguchi S. Fibroblast growth factor (FGF)-23 in patients with primary hyperparathyroidism. European Journal of Endocrinology $200415155-60$

11 Saito H, Maeda A, Ohtomo S, Hirata M, Kusano K, Kato S, Ogata E, Segawa H, Miyamoto K \& Fukushima N. Circulating FGF-23 is regulated by 1alpha,25-dihydroxyvitamin D3 and phosphorus in vivo. Journal of Biological Chemistry $2005 \mathbf{2 8 0}$ 2543-2549.

12 Tebben PJ, Singh RJ, Clarke BL \& Kumar R. Fibroblast growth factor 23, parathyroid hormone, and 1alpha,25-dihydroxyvitamin D in surgically treated primary hyperparathyroidism. Mayo Clinical Proceedings 200479 1508-1513.

Received 2 May 2005

Accepted 16 September 2005 